DEPÓSITO LEGAL ZU2020000153

Esta publicación científica en formato digital

es continuidad de la revista impresa

ISSN 0041-8811

E-ISSN 2665-0428

Revista

de la

Universidad

del Tunlia

Fundada en 1947

por el Dr. Jesús Emrique Lossada

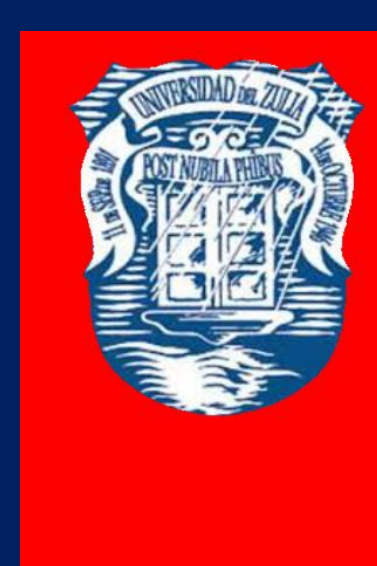

Ciencias

Sociales

y Arte

Aกัต 11 No 31

Septiembre - Diciembre 2021

Tercera ípoca

Maracailbo-Venezuela 


\title{
Plan estratégico de una empresa de almacenes del Perú
}

\author{
Lear Scoth Wong Altamirano * \\ Carlos Alberto Ríos Campos** \\ Juan Alberto Avalos Hubeck *** \\ Nemesio Santamaría Baldera $* * * *$ \\ Ronald Omar Estela Urbina *****
}

RESUMEN

La investigación se denominó "Plan estratégico de una empresa de almacenes del Perú", el objetivo de la presente investigación fue proponer un plan estratégico para una empresa de almacenes del Perú. La investigación se realizó mediante el diseño descriptivo - propositivo; se empleó un cuestionario aplicado a una muestra aleatoria de los trabajadores de la empresa de almacenes. El instrumento consta de 33 ítems divididos en siete dimensiones, tres de planificación estratégica: Formulación - Planificación, Implementación - Gestión y Evaluación - Control; y cuatro administraciones comerciales: Identificación, Organización, Ejecución y Control, categorizados por la escala Likert. La fiabilidad del instrumento estuvo determinada por el alfa de Cronbach, el cual arrojó un resultado de 0.95. La población considerada para el diagnóstico fue de 61 trabajadores de la empresa de almacenes, de los cuales 53 son muestra de estudio. Con los resultados obtenidos del cuestionario, se determinó que los trabajadores tienen poco conocimiento del plan estratégico y la gestión empresarial. Por lo tanto, se planteó proponer un plan estratégico para la mejora de la gestión empresarial. PALABRAS CLAVE: Plan estratégico, gestión empresarial, almacén, Perú.

* Investigador de mercado. Universidad Nacional Pedro Ruiz Gallo. E-mail: lear550@gmail.com

** Docente universitario. Universidad César Vallejo. E-mail: carlosrioscampos@gmail.com

*** Docente de Contabilidad, Universidad Politécnica Amazónica. E-mail: juanavalosh@hotmail.com

**** Docente auxiliar. Universidad Nacional Intercultural Fabiola Salazar Leguía de Bagua. E-mail: nsantamaria@unibagua.edu.pe

**** Docente asociado. Universidad Nacional Intercultural Fabiola Salazar Leguía de Bagua. Email: restela@unibagua.edu.pe 


\section{Strategic plan of a warehouse company in Peru}

ABSTRACT

The research was called "Strategic Plan for a Peruvian Warehouse Company", the objective of this research was to propose a strategic plan for a Peruvian warehouse company. The research was carried out through a descriptive-purposeful design; A questionnaire was applied to a random sample of the workers of the warehouse company. The instrument consists of 33 items divided into seven dimensions, three of strategic planning: Formulation - Planning, Implementation - Management and Evaluation - Control; and four commercial administrations: Identification, Organization, Execution and Control, categorized by the Likert scale. The reliability of the instrument was determined by Cronbach's alpha, which yielded a result of 0.95 . The population considered for the diagnosis was 61 workers from the warehouse company, of which 53 are a study sample. With the results obtained from the questionnaire, it was determined that the workers have little knowledge of the strategic plan and business management. Therefore, it was proposed to propose a strategic plan to improve business management.

KEY WORDS: Strategic plan, business management, warehouse, Peru.

Introducción

La planificación estratégica se ha convertido hoy en una necesidad, tanto para las empresas como para las IES, más allá de la interpretación de sus falacias y periodos de estancamiento (Galarza \& Almuiñas, 2015).

La planificación estratégica es uno de los componentes o funciones de la dirección estratégica y al mismo tiempo, un proceso dinámico y sistemático que contribuye en la supervivencia y crecimiento de una institución (Quintero et al., 2015).

Las tecnologías de almacenes permiten tener una gestión efectiva y planificada de los recursos que una empresa tiene (materiales e información). Cuando contienen información, existe una necesidad de poseer un sistema de almacenamiento de información (TalamanteLugo et al., 2019).

De acuerdo a la tendencia de las grandes corporaciones que están adoptando una serie de estrategias (estas pueden ser reducción en los precios de venta, promociones, valor 
REVISTA DE LA UNIVERSIDAD DEL ZULIA. 3é época. Año $11 \mathrm{~N}^{\circ}$ 31, 2020

Lear Scoth Wong Altamirano et al /// Plan estratégico de una empresa de almacenes del Perú, 26-38

DOI: http://dx.doi.org/10.46925//rdluz.31.03

agregado, cambio de envases y otras), en las cuales se busca el beneficio de alcanzar una mayor rentabilidad estable en el tiempo o abarcar una mayor participación de mercado, entonces conociendo esta problemática es que comienza la necesidad de afrontarse a los cambios que lleva la globalización, indistintamente de la actividad de la organización. Respecto al rubro de bebidas no alcohólicas, existe una tendencia por consumir bebidas más saludables a nivel global, esto conlleva a que las corporaciones estén obligadas a expandir su portafolio de bebidas, teniendo menor consideración a sus bebidas bandera como Pepsi, Coca Cola o Big Cola. Es por esto que el negocio de bebidas no alcohólicas es un mercado altamente competitivo, debido a que el comercio ya no solo se ha vuelto un tema solamente de compra y venta, si no a la introducción de una serie de estrategias para que el consumidor elija, aunque sea una de las marcas que las compañías tienen en el mercado, siendo la competitividad ya no táctica, si no altamente estratégica, que en el tiempo dará los resultados buscados.

Este marketing genera que mucha gente consuma las bebidas sin necesitarlas en realidad. Además, su fácil acceso estimula las ventas, ya que pueden encontrarse desde en los supermercados hasta en la bodega más cercana (Agramonte \& Ronceros, 2016).

Posicionarse en el mercado es la meta de toda industria; para lograr este objetivo necesita tener ventajas competitivas en base a la calidad, el precio, la innovación, entre otros. Para tener productos de buena calidad un requisito primordial es la calidad de su materia prima; en la producción de bebidas gaseosas la principal materia prima es el agua (Vásquez, 2018).

En el Perú el mercado de bebidas no alcohólica en el trascurso de estos tres años ha tenido una sucesión de reformas significativas respecto a las embotelladoras y distribuidoras, tal es el caso de Ambev Perú a CBC Peruana en el año 2015, teniendo una alianza estratégica, también en este año la corporación Lindley Perú realiza una unión con Arca Continental, y también recientemente la adquisición de la embotelladora Don Jorge por parte del Grupo AJE, debido a esta sucesión de cambios para fortalecer e incrementar la participación de las operaciones de las compañías de bebida, ofrecen la oportunidad de un dinamismo respecto a la competitividad dentro del mercado peruano.

Cada marca indica la composición nutricional de distinta manera... Por ello, es importante que en Perú la información también se comunique de forma sencilla y clara. 
REVISTA DE LA UNIVERSIDAD DEL ZULIA. 3é época. Año $11 \mathrm{~N}^{\circ}$ 31, 2020

Lear Scoth Wong Altamirano et al /// Plan estratégico de una empresa de almacenes del Perú, 26-38

DOI: http://dx.doi.org/10.46925//rdluz.31.03

Creemos que recibir esta información en forma estandarizada, es un derecho de los consumidores (Azañedo et al., 2018).

En la empresa de almacenes del Perú, siendo una compañía familiar, fue fundada en el año 2002, comenzó sus funciones en la rúbrica de comercializadora y distribuidora de abarrotes, en el 2005 inicia en el rubro de bebidas con la venta y reparto del portafolio de las marcas de Ambev Perú S.A., tales como Brahma, Corona y las marcas de Pepsico bebidas, en el año 2015, CBC Peruana S.A. en conjunto con AB Inbev, comienza sus funciones en el Perú, continuando con el fabricación y distribución exclusivo de las marcas de Pepsico bebidas, dentro de ellas tenemos a Pepsi, 7up, Evervess, San carlos (Aquafina), Gatorade, Concordia, Triple kola, además ingresa con sus marcas propias como Petit, Frutaris, Jugazzo que pertenecen la gamma de Beliv.

La compañía CBC Peruana, proveedora desde el inicio de sus operaciones está en búsqueda de incrementación en la participación de mercado de bebidas no alcohólicas, de acuerdo a ello viene introduciendo y evaluando la mejora de sus procesos comerciales, inyectando una cultura empresarial que coloque en un estándar a sus principales distribuidores, también el logro de sus diferentes indicadores comerciales, uno de los distribuidores es almacenes del Perú, logró en el año 2016 resultados favorables y satisfactorios, mientras que en los últimos años, los diferentes cambios del mercado que se ha vuelto altamente competitivo, el factor climático, la falta de gestión en buscar la oportuna reacción para revertir los resultados, vienen alejando cada día de los objetivos trazados.

Hasta el año 2016 la empresa de almacenes del Perú, tenía dentro de su portafolio la categoría de cerveza, la cual podríamos decir que era un considerable volumen en la participación con respecto a las ventas, a partir del año 2017 comenzaron a introducir en los sitios de expendio nuevas categorías como Still Drink (aguas saborizadas), energizantes y néctar, sin embargo este nuevo portafolio la empresa almacenes del Perú, no ha logrado alcanzar los resultados obtenidos por la comercialización de la categoría cerveza.

Por ellos CBC Peruana S.A., ha comenzado a implementar una serie de estrategias de marketing, este plan consta de con la exhibición en los puntos de venta (PDV), colocación de Racks (anaqueles de exhibición), equipos de frío (refrigeradoras para el enfriamiento de las bebidas), pintado de fachada, merchandising dentro de los puntos de venta, también acciones 
REVISTA DE LA UNIVERSIDAD DEL ZULIA. 3é época. Año 11 N 31, 2020

Lear Scoth Wong Altamirano et al /// Plan estratégico de una empresa de almacenes del Perú, 26-38

DOI: http://dx.doi.org/10.46925//rdluz.31.03

comerciales como descuentos y/o promociones y la colocación de un portafolio prioritario en la cual corresponde a la venta de una lista de productos principales en cada punto de venta.

Es por ello que el dinamismo en la actualidad en el rubro de bebidas no alcohólicas la empresa de almacenes del Perú, tiene la necesidad de trabajar a la altura las competencias estratégicas actuales, es por eso que se razona la presente proposición de un plan estratégico para aumentar la gestión empresarial de almacenes del Perú.

\section{Metodología}

El tipo de investigación fue descriptivo, pues detalla los tipos y los perfiles de las agrupaciones y objetos que se sometan para el examen. Calcula datos de forma individual o vinculada entre las variables a trabajar (Hernández et al., 2010) y propositivo, porque, usa grupo de herramientas y procesos con el fin de establecer y solucionar dificultades (Hernández et al., 2010).

El diseño de investigación fue transversal con corte en el tiempo, porque recolecta datos en un determinado momento (Hernández et al., 2010). Descriptiva, ya que se describirá el escenario actual que tiene la empresa con relación a la gestión. La investigación es de característica descriptiva, de acuerdo a Hernández et al (2010) tiene como fin investigar la incidencia de las particularidades o niveles de una o más variables en una población, es por ello que el caso en estudio se representa en la situación actual que tiene la compañía como objeto de estudio y que requiere para su mejora mediante la proposición de un plan estratégico. Propositiva, debido a que se elaboró una propuesta de plan de estratégico para mejorar la gestión empresarial en la empresa. La investigación propositiva según Hernández et al (2010) conquista de cómo realmente debería ser las cosas para lograr fines y funcionar convenientemente. Para el caso de estudio, a partir del estudio descriptivo se identificó el requerimiento y se planteó una solución de acuerdo de la propuesta de un plan estratégico.

Según Hernández et al (2010), lo que se trabaja en la investigación no experimental es observar fenómenos tal como se dan en su contexto natural, para posteriormente analizarlos. El diseño de investigación del cual se ha hecho uso es el diseño de carácter no experimental, ya que no se someterá a ninguna prueba. 
2. Resultados

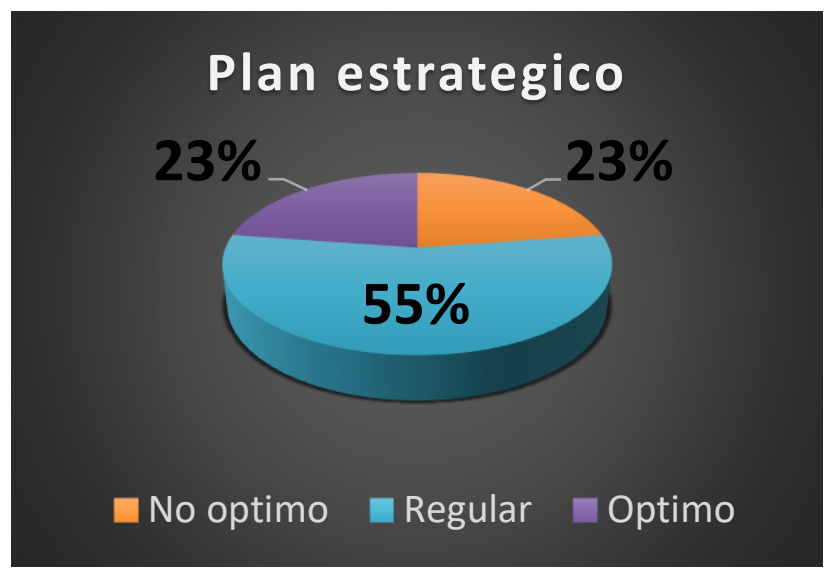

Figura 1. Nivel de planeamiento estratégico en la empresa de almacenes del Perú Fuente: Elaboración propia

Se evidenció que el nivel más predominante es el regular conocimiento del plan estratégico con 55\%, seguido de los niveles óptimos y no óptimos con 23\%, también podemos decir que el conocimiento del plan estratégico es muy disperso de acuerdo que no está bien definido por los colaboradores de la empresa de almacenes del Perú.

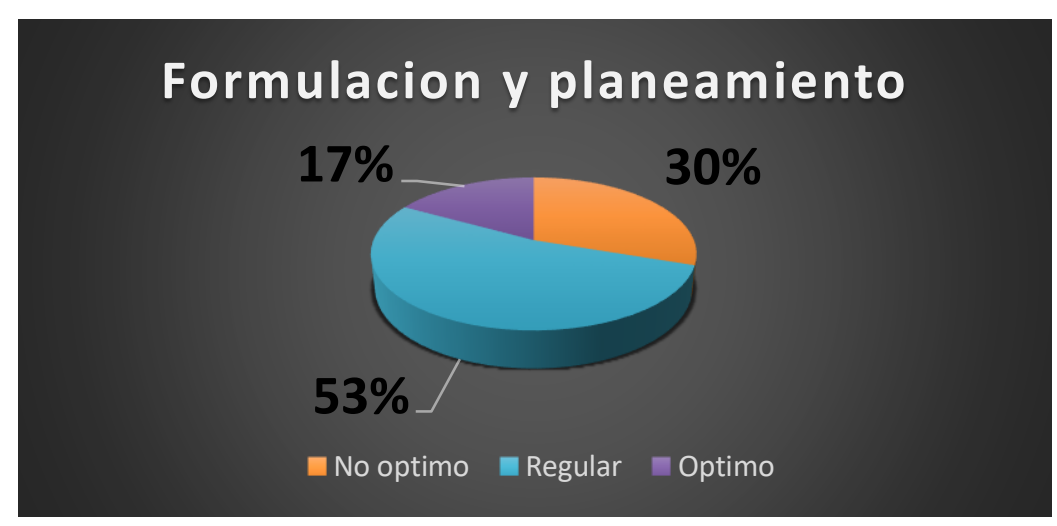

Figura 2. Nivel de planeamiento estratégico de la empresa de almacenes del Perú en la dimensión formulación y planeamiento. Fuente: Elaboración propia

Se observó que el 53\% de los colaboradores tienen un conocimiento regular de la dimensión formulación y planeamiento del plan estratégico, mientras que también evidenciamos un 30\% de los colaboradores que tiene un nivel no óptimo de conocimiento de la dimensión, mientras que un $17 \%$ tiene un nivel óptimo, podemos evidenciar una falta de 
REVISTA DE LA UNIVERSIDAD DEL ZULIA. 3a época. Año 11 N 31, 2020

Lear Scoth Wong Altamirano et al /// Plan estratégico de una empresa de almacenes del Perú, 26-38

DOI: http://dx.doi.org/10.46925//rdluz.31.03

conocimiento en la dimensión ya que existe una dispersión y un porcentaje considerable en los niveles bajos.

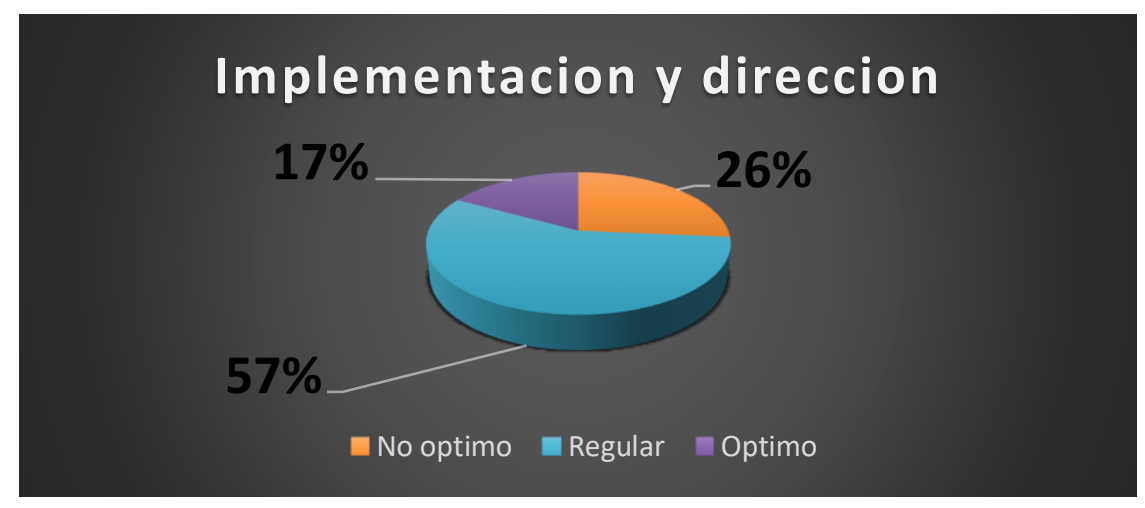

Figura 3. Nivel de planeamiento estratégico de la empresa de almacenes del Perú en la dimensión implementación y dirección. Fuente: Elaboración propia

Se evidenció que el nivel de conocimiento sobre la dimensión implementación y dirección con mayor porcentaje es regular con $57 \%$, seguido de un $26 \%$ que tiene un conocimiento no óptimo y sólo un 17\% de los colaboradores tiene un conocimiento óptimo, es claro notar una deficiencia del conocimiento de los análisis PEST y matriz FODA, es demasiado bajo.

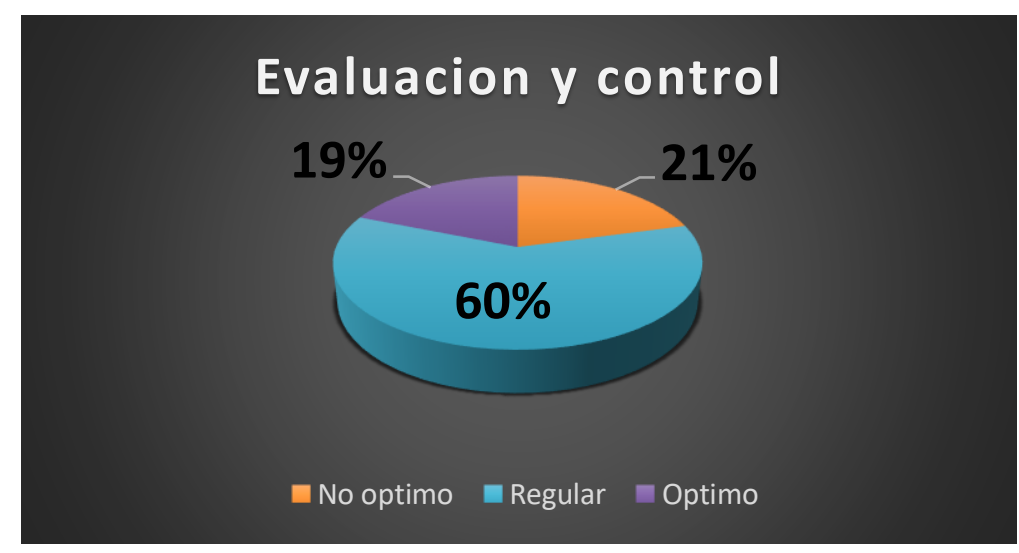

Figura 4. Nivel de planeamiento estratégico de la empresa de almacenes del Perú en la dimensión evaluación y control. Fuente: Elaboración propia

Se evidenció que un $60 \%$ de conocimiento regular en la dimensión mientras que un $21 \%$ en el nivel no óptimo y 19\% en el nivel óptimo, en cuanto a la dimensión evaluación y control 
REVISTA DE LA UNIVERSIDAD DEL ZULIA. 3a época. Año 11 N³1, 2020 Lear Scoth Wong Altamirano et al /// Plan estratégico de una empresa de almacenes del Perú, 26-38

DOI: http://dx.doi.org/10.46925//rdluz.31.03

se tiene un porcentaje considerable en el nivel de conocimiento regular en la cual los colaboradores tienen estimado que la evaluación y control aportará para la mejora del plan estratégico.

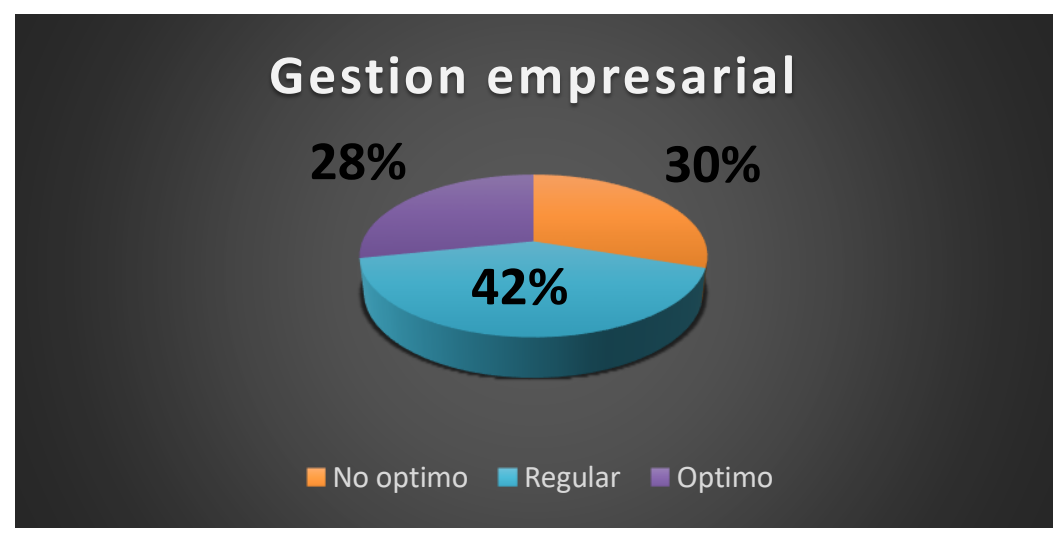

Figura 5. Nivel de gestión empresarial de la empresa de almacenes del Perú Fuente: Elaboración propia

Se evidenció que un porcentaje alto en el nivel regular en gestión empresarial con $42 \%$ seguido de 30\% en el nivel no óptimo y $28 \%$ en el nivel óptimo, podemos decir que el conocimiento de gestión empresarial en los colaboradores de empresa no está bien definido.

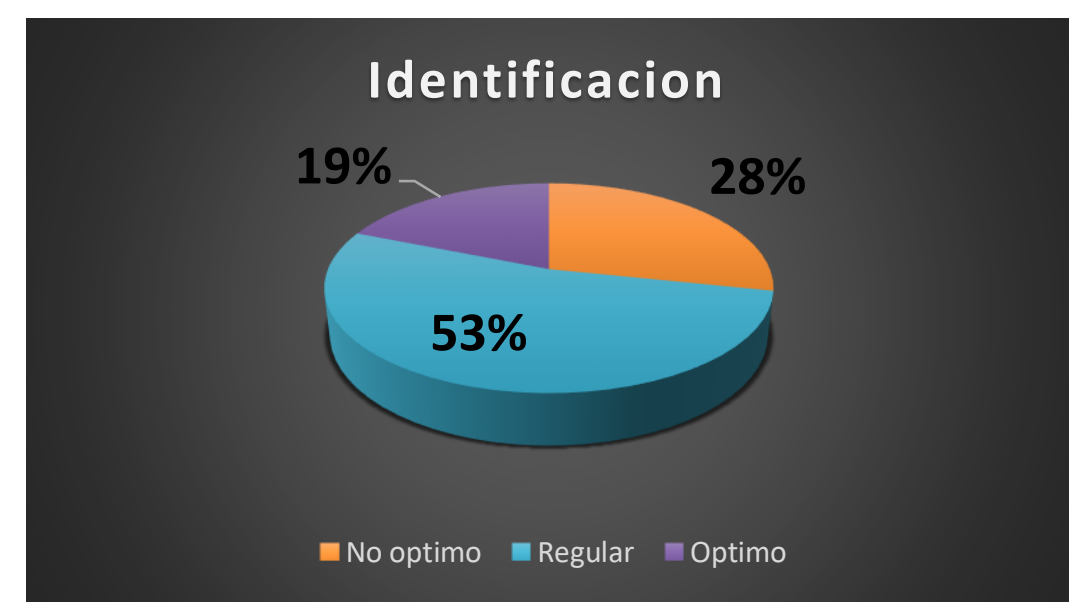

Figura 6. Nivel de gestión de la empresa de almacenes del Perú en la dimensión Identificación. Fuente: Elaboración propia

Se evidenció que un porcentaje de 53\% en el nivel regular de conocimiento en la dimensión identificación, seguido de un 28\% en el nivel no óptimo y 19\% en el nivel no óptimo 
REVISTA DE LA UNIVERSIDAD DEL ZULIA. 3a época. Año 11 N³1, 2020

Lear Scoth Wong Altamirano et al /// Plan estratégico de una empresa de almacenes del Perú, 26-38

DOI: http://dx.doi.org/10.46925//rdluz.31.03

en los colaboradores, lo cual evidencia una dispersión en cuanto a las estrategias, objetivos, las metas, políticas y el plan de acción.

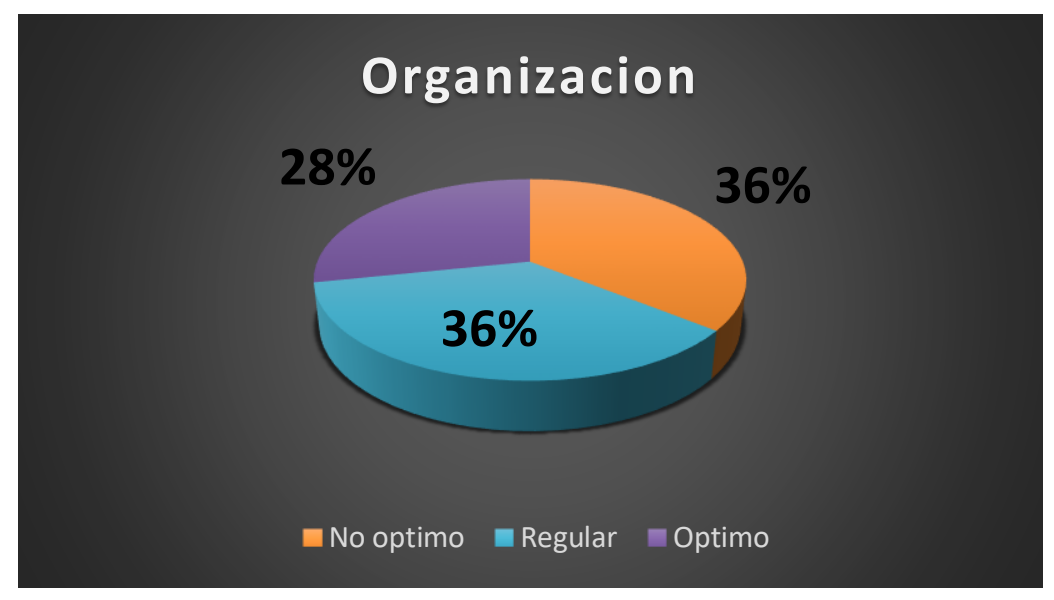

Figura 7. Nivel de gestión de la empresa de almacenes del Perú en la dimensión organización. Fuente: Elaboración propia

Se observó un empate en el porcentaje en los niveles de conocimiento no óptimo y regular con un 36\%, y un 28\% en el nivel óptimo, se evidenció que los trabajadores de la empresa de almacenes del Perú, tienen bajo conocimiento de organización de la empresa. También, carecen de conocimiento en la elaboración de manuales y propuestas de gestión de la empresa.

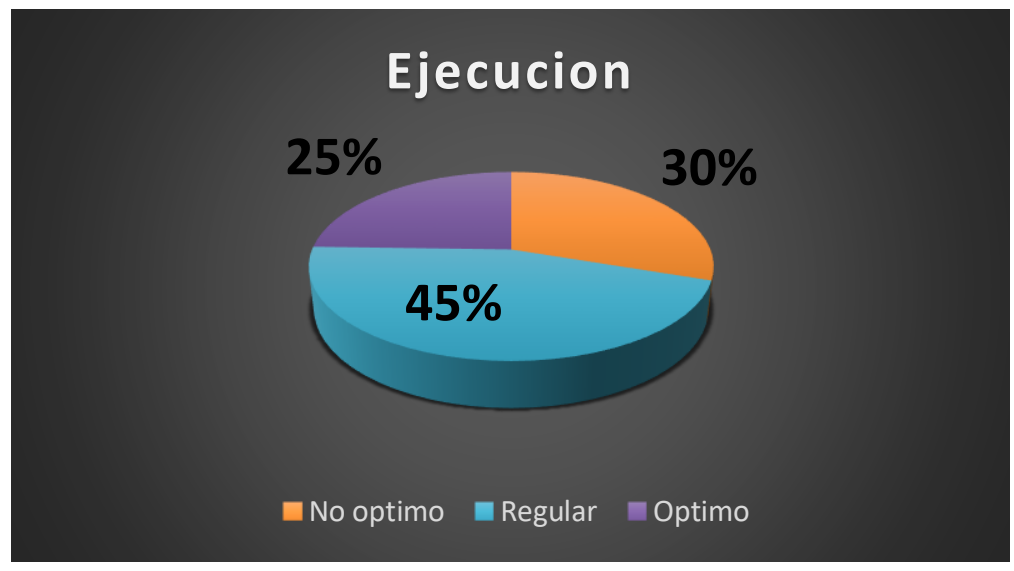

Figura 8. Nivel de gestión de la empresa de almacenes del Perú en la dimensión ejecución. Fuente: Elaboración propia 
REVISTA DE LA UNIVERSIDAD DEL ZULIA. 3e época. Año 11 N 31, 2020

Lear Scoth Wong Altamirano et al /// Plan estratégico de una empresa de almacenes del Perú, 26-38

DOI: http://dx.doi.org/10.46925//rdluz.31.03

En la dimensión ejecución, el 45\% de los colaboradores tienen un conocimiento regular, un 30\% de nivel no óptimo y sólo un 25\% de nivelóptimo, se muestran distanciadas opiniones por la toma de disposiciones, la comunicación y si los altos mandos utilizan un modo interactivo.

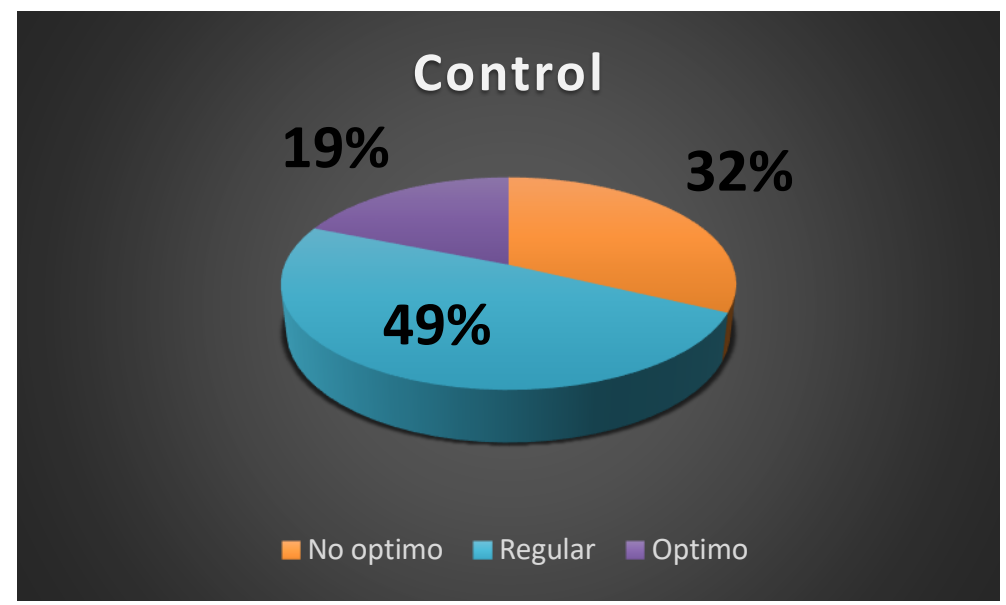

Figura 9. Nivel de gestión de la empresa de almacenes del Perú en la dimensión control Fuente: Elaboración propia

Se observó que en la dimensión control existe un porcentaje considerable en el nivel regular con 49\%, 32\% en el nivel óptimo y solo un 19\% en el nivel óptimo, tienen un bajo conocimiento acerca de la gestión empresarial, pero también afirman que la empresa tiene un sistema de seguimiento de inventarios, finanzas y comercial.

\section{Discusión}

En esta tesis se dio a conocer el horizonte de conocimiento que tienen los colaboradores de la empresa de almacenes del Perú, de las dimensiones del plan estratégico y de la gestión empresarial. Los resultados los mostramos en 13 gráficos que se pueden prestar atención en la división III, en la formulación y planteamiento según D’Alessio (2008) se debe establecer la visión y la misión, los intereses organizacionales, los valores, evaluar los factores internos y externos y determinar los objetivos estratégicos, se recalca un 53\% que tiene un conocimiento bajo sobre el análisis de la empresa, el 30\% conoce un nivel promedio de la visión de la compañía y un 17 \% si conoce suficiente los valores que promueve la compañía. En la implementación y dirección, la gerencia debe desarrollar pasos críticos que orienten a 
REVISTA DE LA UNIVERSIDAD DEL ZULIA. $3^{a}$ época. Año $11 N^{\circ}$ 31, 2020

Lear Scoth Wong Altamirano et al /// Plan estratégico de una empresa de almacenes del Perú, 26-38

DOI: http://dx.doi.org/10.46925//rdluz.31.03

organizar y coordinar las estrategias internas y externas (D'Alessio, 2008), se detalla que la gran parte de colaboradores conoce de manera muy baja respecto a un análisis FODA, PEST, la estructura de un plan estratégico y los procedimientos que tiene la compañía a largo de los plazos. Sobre la evaluación y control según D’Alessio (2008) se debe cerrar los detalles que se encuentren entre lo planeado y lo ejecutado, se plantea acciones de supervisión, se observa que gran parte de los colaboradores conoce medianamente los indicadores que tienden a ser estimados para el cumplimento de los objetivos, que el plan actual es óptimo para la compañía, además que el plan estratégico mejora la gestión de la empresa y que la compañía evalúa la ejecución de un plan.

De acuerdo a la gestión empresarial en sus dimensiones, la forma de diseñar el futuro y hacer que suceda (Prieto, 2011), se puede observar que el 42\% precisa un nivel regular en conocimiento de la dimensión y un 30\% define como poco conocimiento, con respecto a la identificación, es la gestión de los requerimientos por satisfacer, los objetivos, las estrategias, las políticas y los procedimientos (Luna, 2014). Se puntualiza la organización que la gran parte de los colaboradores no han participado en la producción de manuales y proposiciones de gestión, desconoce las partes de la gestión empresarial y no tienen un organigrama. Según Luna (2014) en la ejecución se debe utilizar un liderazgo capaz de informar y motivar para así actuar por persuasión, se detalla que el 36\% conoce regular y no optimo la dimensión, seguido de que el $28 \%$ conoce bastante que la toma de decisiones es rápida y pertinente. Se observa el poco conocimiento sobre el control seguido de la gestión y los itinerarios con un $49 \%$ y $32 \%$ respectivamente, mientras que el 19\% conoce que la compañía cuenta con un sistema de control en sus ventas e inventarios.

Por lo que, en base a los resultados se deriva que se debe mejorar el horizonte de conocimiento del plan estratégico en sus aspectos, además del conocimiento en la gestión empresarial de sus 4 aspectos: identificación, organización, ejecución y control, para ello se plantea la propuesta de un plan estratégico para una empresa de almacenes del Perú: un 28\% afirma esta medida, mientras que un $42 \%$ lo cree en un nivel promedio y solo el $30 \%$ considera de manera baja esta medida.

Para una organización es vital ya que sobrelleva a ejecutar un análisis de la situación general de la compañía, a establecer qué es lo que busca la organización, visión, cual es la intención de la organización, misión, para un adecuado y ético desempeño, a ejecutar un 
REVISTA DE LA UNIVERSIDAD DEL ZULIA. 3e época. Año 11 N 31, 2020

Lear Scoth Wong Altamirano et al /// Plan estratégico de una empresa de almacenes del Perú, 26-38

DOI: http://dx.doi.org/10.46925//rdluz.31.03

examen interno (FODA) y externo (PEST), para que después de este trabajo hagamos planes de acción, estrategias para el logro de objetivos y que en el transcurso estos planes se evalúen y fiscalicen. Es por esto que la gestión empresarial es la que debe identificar, organizar, ejecutar y controlar a los trabajadores, en este caso de la empresa de almacenes del Perú.

\section{Conclusiones}

La propuesta de un plan estratégico para la empresa de almacenes del Perú, es factible dado que los resultados del cuestionario aplicado indican que sus trabajadores no tienen un conocimiento despejado acerca de estas herramientas gerenciales, como sí lo son el plan estratégico y la gestión empresarial.

Acerca del diagnóstico de la empresa de almacenes del Perú, los resultados de la encuesta aplicada indican una alta dispersión entre los conocimientos de la visión, los valores, las metas, objetivos y estrategias que aplica la empresa de almacenes del Perú. Se debe enseñar a cada uno de sus colaboradores, lo que se quiere y busca lograr en un futuro y cuál es la finalidad de la empresa.

Se identificó que los colaboradores de la empresa de almacenes del Perú, no tienen una participación en la elaboración del análisis PEST y la matriz FODA por lo que se debe buscar una participación activa de los colaboradores para que conozcan la situación actual de la empresa y así concientizarlos para el logro de objetivos.

Se identificó una alta dispersión entre los planes de acción de un área específica y los planes de corto, mediano y largo plazo de la empresa de almacenes del Perú, por lo que se debe ejecutar una comunicación efectiva y persuasiva a todos sus colaboradores para alcanzar las metas establecidas (Lachira et al., 2020; Vásquez et al., 2019).

En cuanto al diseño de evaluaciones de control a los planes de acción, se debe plantear acciones de supervisión como el control semanal, mensual, trimestral, semestral y anual y así corregir los detalles entre lo planeado y lo ejecutado.

\section{Referencias}

Agramonte, D. \& Ronceros, L. (2016). Estudio para la instalación de una planta productora de bebida energética gasificada a base de maca negra, hoja de coca y arándano. Ingeniería Industrial, núm. 34, enero-diciembre, pp. 177-194. Universidad de Lima. Lima, Perú 
REVISTA DE LA UNIVERSIDAD DEL ZULIA. 3e época. Año 11 N 31, 2020

Lear Scoth Wong Altamirano et al /// Plan estratégico de una empresa de almacenes del Perú, 26-38

DOI: http://dx.doi.org/10.46925//rdluz.31.03

Azañedo, D., Saavedra-Garcia, L. \& Bazo-Alvarez, J. (2018). ¿Son menos dañinas las gaseosas sin azúcar? un análisis de la información nutricional en dos ciudades peruanas. Rev Peru Med Exp Salud Publica. 35(1):164-6. doi: 10.17843/rpmesp.2018.351.3555.

D’Alessio, F. (2008). El proceso estratégico. Un enfoque de gerencia. Mexico: Pearson Educación de México S.A.

Galarza, J. \& Almuiñas, J. (2015). La gestión de los riesgos de planificación estratégica en las instituciones de educación superior. Revista Cubana de Educación Superior, 34(2), 45-53. Recuperado de http://scielo.sld.cu/scielo.php?script=sci_arttext\&pid=S0257$43142015000200005 \& \operatorname{lng}=$ es\&tlng=es.

Hernández, R., Fernández, C., \& Baptista, M. (2010). Metodología de la Investigación. 5ª ed. Mexico: Mcgraw-Hill / Interamericana Editores, S.A. de C.V.

Lachira Estrada, Diego Salvador; Luján Vera, Priscila Estelita; Mogollón Taboada, Marlón Martín; Silva Juárez, Raquel (2020). La comunicación asertiva: una estrategia para desarrollar las relaciones interpersonales, Revista Latinoamericana de Difusión Científica, 2 (3), 72 82. DOI: https://doi.org/10.38186/difcie.23.06

Luna, A. (2014). Administración estratégica. Grupo Editorial Patria. Recuperado de https://books.google.com.pe/books?id=HPHhBAAAQBAJ\&printsec=frontcover\&ssource=gbs _ge_summary_r\&ecad=0\# $\mathrm{v}=$ onepage\& $\& \& f f=$ false

Prieto, J. (2011). Gestión estratégica organizacional. $3^{\mathrm{a}}$ ed. Bogotá: Ecoe Ediciones.

Quintero, J., López, E., \& Rivero, K. (2015). Planeación estratégica con enfoque prospectivo para la editorial "Universo Sur". Revista Universidad y Sociedad [seriada en línea], 7 (3). pp. 160 167. Recuperado de http://rus.ucf.edu.cu

Talamante-Lugo et al. (2019). Use of Storage Technologies to select Knowledge Management Tools and Strategies for M-SMEs. Ingeniare. Revista Chilena de Ingeniería, 27(3), 421-430. https://dx.doi.org/10.4067/S0718-33052019000300421

Vásquez, E. (2018). Reducción de la variabilidad de un proceso de desmineralización de agua por intercambio iónico para la elaboración de bebidas gaseosas. Enfoque UTE, 9(3), 93-105. https://dx.doi.org/10.29019/enfoqueute.v9n3.307

Vásquez Gastelumendi, D.; Ríos Campos, C. A.; Baldera, N. S.; Gutiérrez Valverde, K. S.; Camacho Delgado, F. M.; Aguirre Zaquinaula, I. R.; Estela Urbina, R. O. (2019). Estrategias organizacionales para fortalecer el clima laboral en la Escuela Profesional de Tecnología Médica - Facultad de Ciencias de la Salud de la Universidad de Chiclayo, Revista de la $\begin{array}{lllll}\text { Universidad del } & \text { Zulia, } & 10 & \text { (28), }\end{array}$ https://produccioncientificaluz.org/index.php/rluz/article/view/30602 